\title{
Editorial
}

\section{Targeting angiogenesis: genetic intervention which strikes at the weak link of tumorigenesis}

Tumour angiogenesis has become the focus of intense interest as a potential target for novel cancer therapies. A new generation of anticancer drugs such as TNP-470 (AGM-1470) is already in early clinical trial and shows a potent antitumour activity through inhibition of angiogenesis. In mouse models, angiostatin, an endogenous angiogenesis inhibitor, has also been shown not only to suppress primary tumour growth but also to produce long-term dormancy of metastasis. ${ }^{1}$ These results encourage us to consider new strategies for cancer gene therapy. What is the advantage of targeting angiogenetic endothelial cells in tumour tissue rather than targeting tumour cells themselves? First, we can expect a broad spectrum of antitumour activity instead of different levels of tolerance against conventional types of antitumour drugs. Second, tumour cells may be destroyed without the induction of tumour cell-associated drug resistance. Third, damage to only a single vessel might kill millions of tumour cells simultaneously.

Angiogenesis is an essential process during development and wound repair, but is also involved in the pathology of various diseases including cancer. Tumour growth and metastasis depend on angiogenesis. Without neovascularization, cells in prevascular tumours or metastases that may be replicating rapidly reach equilibrium with their rate of death. The critical balance between positive and negative regulators of angiogenesis in the local environment is important for microvessel formation. For instance, the inevitable hypoxia within an expanding tumour applies a selective pressure on the malignant cells, such that those exhibiting reduced apoptotic potential tend to survive and the expression of certain genes like vascular endothelial growth factor (VEGF) is modulated by endogenous factors (such as mutant ras expression and p53 inactivation $)^{2}$ and exogenous influences (such as hypoxia). VEGF has a variety of functions including promoting endothelial cell proliferation, increasing vascular permeability and inducing procoagulant factors and matrix metalloproteinases. It is transcriptionally controlled (at least in part) by a hypoxiainducible factor and thus hypoxia triggers overexpression of VEGF. The VEGF produced binds to two high affinity receptors expressed exclusively on endothelial cells, VEGF-R1 (flt-1) and VEGF-R2 (flk-1/KDR). Interestingly, the newer members of the VEGF family VEGF-B and VEGF-C are not up-regulated by these triggers nor by hypoxia, but rather in the case of VEGF-B by serum fac- tors such as platelet-derived growth factor (PDGF), epidermal growth factor (EGF) and transforming growth factor- $\beta .^{3}$

There are three ways to change the balance of angiogenesis regulators to inhibit tumour neovascularization: (1) to suppress positive regulators; (2) to induce negative regulators; and (3) to suppress receptor expression on endothelial cells.

Several studies have been reported using nongenetic approaches such as neutralizing antibodies against ligands or receptors of positive regulators of angiogenesis. Administration of a monoclonal antibody to VEGF inhibited the growth of subcutaneous human xenograft tumours and limited the number and size of hepatic metastases in athymic mice. ${ }^{4}$ More recently, a blocking antibody to VEGF-R2 has been demonstrated to produce a reduction in angiogenesis and malignant cell invasion, although tumour cell proliferation was unaffected. ${ }^{5}$ The agent TNP-470 (O-chloracetylcarbamoyl fumagillol, also named AGM-1470) inhibits endothelial cell proliferation at concentrations that are inactive against other cell types, whereas cytotoxic reagents like cisplatin and doxorubicin do not show such differential effects. ${ }^{6}$ TNP-470 is presently in use in phase I and phase II cancer trials.

What is the advantage of genetic approaches for antiangiogenesis? Arguably, genetic approaches would be preferable as they could allow more efficient, long-term effects compared with synthetic inhibitors which need to be administered chronically to maintain any benefit, or antibodies that are often destroyed on repeat administration by anti-idiotype antibodies. Furthermore, it might be possible to force cancer cells themselves to produce anti-angiogenic factors, that could more effectively change the local balance to promote the disruption of tumour-associated vessels. However, so far few therapeutic studies using a genetic approach for anti-angiogenesis have been carried out.

Is it really possible to target tumour vasculature or angiogenic endothelial cells without disturbing normal endothelial cells? Which angiogenic process or structure is the most effective target for therapy?

Anti-sense and ribozyme strategies against angiogenic factors have achieved some success in suppressing angiogenesis in cancer therapy. Ellis et $a l^{7}$ demonstrated that a colon carcinoma cell line transfected with an antisense construct to $c$-src blocked the tyrosine kinase activity that is the normal pathway of activation by VEGF. It was also reported that reduction of fibroblast growth factor (FGF)binding protein (FGF-BP) using specific ribozymes reduced the level of biologically active basic FGF (FGF2), and the growth and angiogenesis of mouse model xeno- 
grafts decreases in parallel with the reduction of FGFBP. ${ }^{8}$ Pleiotrophin is a secreted polypeptide growth factor that is a mitogen for endothelial cells, epithelial cells and fibroblasts. Pleiotrophin RNA and protein levels were reduced by transfecting melanoma cells with pleiotrophin-targeted ribozymes. In vitro there was no effect on melanoma cell growth, but in vivo tumour growth and angiogenesis were reduced in parallel with the pleiotrophin level. ${ }^{9}$

Molecules such as platelet factor 4 (PF4), thrombospondin-1 (TSP-1), tissue inhibitors of metalloproteinases (TIMPs), angiostatin and endostatin are endogenous inhibitors of endothelial cell proliferation and migration. Tanaka et $a l^{10}$ used adenoviral and retroviral vectors to deliver the gene for PF4 to RT2-glioma cells and demonstrated that they were able to inhibit the proliferation of adjacent endothelial cells in vitro. When the same cells were used to seed intracranial tumours in mice and the PF4 gene was delivered by adenoviral vector there was reduced tumour angiogenesis and prolonged survival, making this a promising strategy. TSP-1, a homotrimetric, multifunctional glycoprotein, is one of the major secreted proteins of human platelets and an extracellular component of a variety of cells including vascular endothelial cells. Transfection of TSP-1 cDNA into human breast cancer cells successfully reduced primary tumour growth, metastatic potential and angiogenesis. ${ }^{11}$ Tissue inhibitors of matrix metalloproteinases (TIMPs), especially TIMP-2, have been shown to suppress tumour metastasis and angiogenesis. TIMP-2 has a high specificity for MMP-2 (gelatinase A) and can inhibit its activity to degrade extracellular matrix (ECM) components. In an in vivo analysis of melanoma cell lines, TIMP-2-transfected clones showed reduced levels of blood vessel formation, and in vitro conditioned media from TIMP-2 transfectants showed diminished induction of endothelial cell migration and invasion. ${ }^{12}$ Angiostatin is a fragment of plasminogen that has been reported to be secreted by the primary tumour and which appears to maintain metastases in a dormant state by inhibiting angiogenesis. It has been shown that transfection of the cDNA encoding angiostatin in vivo can suppress primary and metastatic fibrosarcoma tumour growth. ${ }^{1}$ Endostatin consists of the C-terminal fragment of collagen XVIII. It specifically inhibits endothelial cell proliferation. Recombinant mouse endostatin administered to mice with Lewis lung carcinoma metastases resulted in a highly significant reduction of metastatic growth compared with mice treated with saline. ${ }^{13}$

In this issue of Gene Therapy, another system has been targeted for genetic intervention to inhibit tumour angiogenesis. ${ }^{14}$ The amino-terminal fragment of urokinase (ATF) has been shown to have potential as a novel inhibitor of tumour neovascularisation. This secreted molecule can bind to urokinase receptor (uPAR) on the cell surface but lacks the proteinase activity of full-length uPA. Urokinase (uPA) plays an important role in pericellular proteolysis during cell migration, tissue remodelling and angiogenesis not only by physiological activation of plasminogen to plasmin but also initiation of intracellular signalling even though this receptor is linked to the plasma membrane only by a glycosyl phosphatidyl inositol (GPI) anchor. Several reports have shown that up-regulation of UPAR enhances migration not only by tumour cells but also by endothelial cells leading to neovascularization.
Thus, inhibition of the $\mathrm{uPA} / \mathrm{uPAR}$ complex is a potentially powerful strategy for cancer gene therapy. Li et al ${ }^{14}$ show suppression of angiogenesis-dependent tumour growth using adenovirus-mediated gene delivery of murine ATF in xenograft and syngenic murine models and importantly, they further demonstrate that tumour cell dissemination is also inhibited by the same system. These findings suggest that targeting therapy against the uPA/uPAR complex on both the tumour cells and angiogenic endothelial cells may have real potential in the management of cancer.

Another strategy for anti-angiogenesis is disruption of microvessel formation by directly targeting the angiogenic endothelial cells of the tumour vasculature. A few targets which are specifically expressed on endothelial cells forming new vessels have already been described including integrin $\alpha_{v} \beta_{3}$, Tie2, vascular endothelialcadherin (VE-cadherin), E-selectin and tissue factor (TF). Integrin $\alpha_{v} \beta_{3}$ can be detected on angiogenic endothelial cells in physiological and pathological angiogenesis, but not on quiescent endothelial cells, and is also expressed on various types of cells including tumour cells. This integrin mediates binding to extracellular matrix components such as vitronectin, fibronectin, von Willebrand factor and fibrinogen. Antagonists of $\alpha_{v} \beta_{3}$ such as specific monoclonal antibody and the cyclic RGD peptide have been demonstrated to inhibit tumour growth and angiogenesis because of interference with adhesion-dependent signals causing apoptosis of angiogenic endothelial cells by suppression of p53 activity and induction of p $21^{\mathrm{WAF} 1 / \mathrm{CIP} 1} .^{15}$ Recently it has been reported that exposure of human endothelial cells to TNF and IFN- $\gamma$ suppresses $\alpha_{v} \beta_{3}$ activation leading to a decrease in endothelial cell adhesion and survival. ${ }^{16}$ Genetic approaches using either an anti-sense strategy to reduce the expression of $\alpha_{v} \beta_{3}$, or a combination of TNF and IFN- $\gamma$ to inactivate $\alpha_{v} \beta_{3}$ might be useful for the selective disruption of angiogenic blood vessels. Tie2 is a novel receptor tyrosine kinase that is expressed predominantly in angiogenic endothelial cells. Lin et $a l^{17}$ showed that inhibition of the Tie2 pathway may yield effective anti-angiogenic treatment of cancer. VE-cadherin is exclusively expressed in angiogenic endothelial cells and is located at points of cell-cell contact. Disruption of VE-cadherin by gene targeting or inhibition using a neutralising $\mathrm{mAb}$ inhibits capillary tube formation in mice and an in vitro endothelial cell monolayer model. ${ }^{18}$ E-selectin, a member of the selectin family of adhesion molecules, also participates in angiogenesis. It is clear that angiogenic endothelial cells require cell-cell interaction to sustain a proper configuration for survival, proliferation and differentiation and that adhesion molecules may represent a good target for genetic intervention.

Another potential candidate is tissue factor (TF), a transmembrane glycoprotein which can activate the coagulation zymogen FVII to the activated form FVIIa. The TF/FVIIa complex initiates the coagulation cascade leading to thrombin generation and fibrin deposition. TF is also a member of the class II cytokine receptor superfamily, which includes the interferon (IFN) $\alpha / \beta$ and $\gamma$ receptor, inducing an intracellular signal upon binding of its specific ligand FVII(a). TF plays a crucial role in development of yolk sac vessel formation and is also expressed in angiogenic endothelial cells within tumour tissue. Interestingly, metastatic properties of cancer cells 
were suppressed not only by a neutralising $\mathrm{mAb}$ against TF, but also by FVIIai (antagonist of FVIIa, whose active site is inhibited) in a lung metastasis model. ${ }^{19}$ Our own data suggest that signalling through the TF/FVIIa pathway is involved in specific up-regulation of UPAR gene expression, resulting in enhanced invasive properties of cancer cells. ${ }^{20}$ Inhibition of the TF/FVII(a) complex using genetic approaches to reduce TF expression or induce antagonist of FVII could be an attractive approach to suppress the epithelial-stromal interactions which are critical to angiogenesis and tumour invasion.

Angiogenesis is now one of the most exciting targets for cancer therapy, and genetic intervention is a powerful tool to exploit our developing knowledge of the biological processes involved. We can predict an explosion in gene therapies aimed at angiogenesis as the focus of interest shifts from the cancer cell itself to the epithelialstromal interface, the weak link in tumorigenesis.

T Taniguchi, A Rigg and NR Lemoine Imperial Cancer Research Fund Molecular Oncology Unit Imperial College School of Medicine Hammersmith Campus Du Cane Road, London W12 0NN, UK

\section{References}

1 Cao $\mathrm{Y}$ et al. Expression of angiostatin cDNA in a murine fibrosarcoma suppresses primary tumor growth and produces longterm dormancy of metastases. J Clin Invest 1998; 101: 1055-1063.

2 Mukhopadhyay D, Tsiokas L, Sukhatme VP. Wild-type p53 and v-src exert opposing influences on human vascular endothelial growth gene expression. Cancer Res 1995; 55: 6161-6165.

3 Enholm B et al. Comparison of VEGF, VEGF-B, VEGF-C and Ang-1 mRNA regulation by serum, growth factors, oncogenes and hypoxia. Oncogene 1997; 14: 2475-2483.

4 Warren RS et al. Regulation by vascular endothelial growth factor of human colon cancer tumorigenesis in a mouse model of experimental liver metastasis. J Clin Invest 1995; 95: 1789-1797.

5 Skobe $\mathrm{M}$ et al. Halting angiogenesis suppresses carcinoma cell invasion. Nature Med 1997; 3: 1222-1227.

6 Folkman J. Clinical applications of research on angiogenesis. New Engl J Med 1995; 333: 1757-1763.
7 Ellis LM et al. Down-regulation of vascular endothelial growth factor in a human colon carcinoma cell line transfected with an antisense expression vector specific for c-src. J Biol Chem 1998; 273: 1052-1057.

8 Czubayko $\mathrm{F}$ et al. A secreted FGF-binding protein can serve as the angiogenic switch in human cancer. Nature Med 1997; 3: 1137-1140.

9 Czubayko F, Schulte AM, Berchem GJ, Wellstein A. Melanoma angiogenesis and metastasis modulated by ribozyme targeting of the secreted growth factor pleiotrophin. Proc Natl Acad Sci USA 1996; 93: 14753-14758.

10 Tanaka T et al. Viral vector-mediated transduction of a modified platelet factor 4 cDNA inhibits angiogenesis and tumor growth. Nature Med 1997; 3: 437-442.

11 Weinstat-Saslow DL et al. Transfection of thrombospondin 1 complementary DNA into a human breast carcinoma cell line reduces primary tumour growth, metastatic potential and angiogenesis. Cancer Res 1994; 54: 6504-6511.

12 Valente $\mathrm{P}$ et al. TIMP-2 over-expression reduces invasion and angiogenesis and protects B16F10 melanoma cells from apoptosis. Int J Cancer 1998; 75: 246-253.

13 O'Reilly MS et al. Endostatin: an endogenous inhibitor of angiogenesis and tumor growth. Cell 1997; 88: 277-285.

$14 \mathrm{Li} \mathrm{H}$ et al. Adenovirus-mediated delivery of a uPA/uPAR antagonist suppresses angiogenesis-dependent tumor growth and dissemination in mice. Gene Therapy 1998 (in press).

15 Strömblad $S$ et al. Suppression of p53 activity and p21 WAF1/CIP1 expression by vascular cell integrin $\alpha_{v} \beta_{3}$ during angiogenesis. $J$ Clin Invest 1996; 98: 426-433.

16 Rüegg $C$ et al. Evidence for the involvement of endothelial cell integrin $\alpha_{v} \beta_{3}$ in the disruption of the tumour vasculature induced by TNF and INF- $\gamma$. Nature Med 1998; 4: 408-414.

17 Lin $\mathrm{P}$ et al. Inhibition of tumour angiogenesis using a soluble receptor establishes a role for Tie2 in pathogenic vascular growth. J Clin Invest 1997; 100: 2072-2078.

18 Breier $\mathrm{G}$ et al. Molecular cloning and expression of murine vascular endothelial-cadherin in early stage development of cardiovascular system. Blood 1996; 87: 630-641.

19 Mueller BM, Ruf W. Requirement for binding of catalytically active factor VIIa in tissue factor-dependent experimental metastasis. J Clin Invest 1998; 101: 1372-1378.

20 Taniguchi T, Kakkar AK, Lemoine NR. Enhanced expression of urokinase receptor induced through the tissue factor-factor VIIa pathway in human pancreatic cancer. (Submitted). 